\title{
Bioeconomic modeling of intervention against clinical mastitis caused by contagious pathogens
}

\author{
T. Halasa ${ }^{1}$ \\ The National Veterinary Institute, Technical University of Denmark, 1870 Frederiksberg C, Copenhagen, Denmark
}

\begin{abstract}
The objective of this study was to assess the epidemiologic and economic consequences of intervention against contagious clinical mastitis during lactation. A bioeconomic model of intramammary infections (IMI) was used to simulate contagious spread of Staphylococcus aureus, Streptococcus uberis, and Streptococcus dysgalactiae, and an environmental spread of Escherichia coli IMI in a 100-cow dairy herd during 1 quota year. The costs of clinical IMI, subclinical IMI, and intervention were calculated into the total annual net costs of IMI during lactation per scenario and compared with a default scenario. Input parameter values were based on the scientific literature. The scenarios were $3-\mathrm{d}$ intramammary lactational treatment (default), 5 -d intramammary treatment, 5-d intramammary treatment and 3-d systemic treatment, 3-d intramammary treatment and culling bacteriologically unrecovered clinical IMI cows, and 5-d intramammary treatment and culling bacteriologically unrecovered clinical IMI cows. Sensitivity analysis was conducted on parameter input values. The results showed that interventions including antibiotic treatment combined with culling unrecovered clinical IMI cows resulted in the lowest transmission, number of IMI cases, and persistent subclinical IMI cases. Nonetheless, the high associated costs of culling bacteriologically unrecovered clinical IMI cows made the other scenarios with a long and intensive antibiotic treatment, but without culling, the most cost effective. The model was sensitive to changes to the cure rate of clinical IMI following treatment, but the ranking of the intervention scenarios did not change. The model was most sensitive to the changes to the transmission rate of Staph. aureus. The ranking of the intervention scenarios changed at low transmission rate of this pathogen, in which the default scenario became the most cost-effective scenario. In case of high transmission of contagious IMI pathogens, long and intensive treatment of clinical
\end{abstract}

Received February 27, 2012.

Accepted June 11, 2012.

${ }^{1}$ Corresponding author: tahbh@vet.dtu.dk
IMI should be preceded by strategies that lower the transmission.

Key words: clinical mastitis, treatment, economic model, transmission

\section{INTRODUCTION}

Clinical IMI is a frequent and costly disease in dairy cattle herds (Halasa et al. 2007). It is also a major welfare problem for dairy cows (Broom, 1991). Clinical IMI is usually treated with 3 -d intramammary treatment, but could be extended depending on the severity of the case and the treatment regimen (Barkema et al., 2006). The success of treatment is highly dependent on the causative agent (e.g., McDougall et al., 2007; Bradley and Green, 2009). Several treatment regimens have been suggested, including culling of the infected cows (Steeneveld et al., 2011). Treated, but unrecovered cases could persist as chronic subclinical IMI, amplifying the risk of infection to other herd mates (Halasa et al., 2009). Until recently, several attempts have been made to assess the cost-effectiveness of interventions against clinical IMI, including antimicrobial treatment and culling of IMI cows. For instance, Bar et al. (2008) developed a dynamic programming model to support farmers in decisions related to treatment of clinical IMI. Steeneveld et al. (2011) investigated the effectiveness of cow-specific treatment of clinical IMI cases in short- and long-term antibiotic treatment regimens, supported with systemic antibiotic treatment. Pinzón-Sánchez et al. (2011) estimated the economic consequence of treatment strategies for mild and moderate clinical mastitis cases using decision tree analysis. Heikkilä et al. (2012) used the dynamic programming approach to estimate the costs of clinical IMI taking into account the indirect costs of premature culling of the clinical IMI cases.

Nonetheless, previous assessments of the economic consequence of interventions against clinical IMI have ignored modeling the dynamics of transmission of contagious IMI pathogens between cows at the herd level. When modeling the spread of a contagious infection between individuals, usually a Reed-Frost model (Becker, 1989 ) is used, in which the probability of infection is 
calculated dynamically based on the transmission rate of infection per unit of time, the number of susceptible individuals, and the number of infected individuals (Becker, 1989; Dohoo et al., 2003). This means that the probability of infection is dependent, among other factors, on the number of infected cows within the herd. Thus, when a clinical IMI cow is treated and recovered, it would not only result in direct benefits from, for instance, reducing milk production loss and risk of premature culling, but also in indirect effects from reducing the risk of infection to healthy herd mates. This is expected to decrease the costs of clinical and subclinical IMI at the herd level (Steeneveld et al., 2011). Therefore, previous studies could have underestimated the true effect of interventions against contagious clinical IMI cases, because of ignoring modeling the transmission dynamics of contagious IMI pathogens between cows at the herd level. Thus, economic effects of IMI interventions can be properly studied only when the transmission dynamics of contagious IMI pathogens are modeled. The objective of this study was to estimate the epidemiologic and economic consequence of interventions, including antibiotic treatment and culling, against contagious clinical IMI during lactation using a bioeconomic simulation model incorporating the dynamics of IMI.

\section{MATERIALS AND METHODS}

\section{Model Description}

The bioeconomic model used in this study is a previously published stochastic and dynamic simulation model of IMI with slight modifications to evaluate the economic consequence of intervention against clinical IMI during lactation in 1 quota year. The model simulates the dynamics of pathogen-specific IMI within dairy herds during lactation (Halasa et al., 2009) and the dry period (Halasa et al., 2010). The model was written using Mathematica 6.0 software (Wolfram Research, Champaign, IL). In brief, the dynamics of Staphylococcus aureus, Streptococcus uberis, Streptococcus dysgalactiae, and Escherichia coli were simulated at cow level using a discrete-event simulation model in a 100-cow dairy herd in a milk quota system. Each time period in the model was $2 \mathrm{wk}$. Reed-Frost models were used to model IMI dynamics of contagious pathogens (Staph. aureus, Strep. uberis, and Strep. dysgalactiae) during the lactation, whereas a Greenwood model was used to model IMI dynamics of E. coli. During the dry period, Greenwood models for all 4 pathogens were assumed to represent IMI dynamics. Dry cow therapy was applied to every cow during dry-off.
During lactation, the probability of acquiring an IMI was determined at the beginning of each time period based on the number of pathogen-specific IMI cows in the previous time period, the number of susceptible cows, and a pathogen-specific transmission rate. For $E$. coli IMI, a constant probability of infection was used, based on the cumulative incidence of $E$. coli IMI per 14 cow-days at risk. During the dry period the probability of new IMI per 14 cow-days at risk and the cure of existing IMI at dry off following antimicrobial therapy were implemented as explained previously (Halasa et al., 2010).

Cows were culled due to clinical IMI, subclinical IMI, and other reasons and were replaced by heifers based on the need for new animals to fill the milk quota (Halasa et al., 2009). Culling of clinical IMI cows was changed depending on the simulated scenario as explained in the subsequent section. The calculated difference between the actual and the expected herd-level milk production at each time period was used to decide on the introduction of replacement heifers to fulfill the milk quota (Halasa et al., 2009).

\section{Modeling Intervention Scenarios}

Four scenarios of clinical IMI intervention were modeled and were compared with a default scenario of clinical IMI intervention. The default scenario consisted of the normal 3-d intramammary treatment of clinical IMI cases (Steeneveld et al., 2011). Two scenarios consisted of long-duration antibiotic treatment, 1 with supportive systemic treatment, and 2 scenarios consisted of antibiotic treatment combined with culling the treated, but bacteriologically unrecovered cases. The scenarios were (A) the default scenario, with 3-d intramammary treatment; (B) scenario 1, a clinical IMI case received for 5-d intramammary treatment; (C) scenario 2, a clinical IMI case received for 5-d intramammary treatment combined with 3-d systemic antibiotic treatment; (D) scenario 3, similar to the default scenario, but if the clinical IMI case did not bacteriologically recover (confirmed by bacteriological culture), it was culled during the subsequent time period; and (E) scenario 4 , similar to scenario 1 , but if the clinical case did not bacteriologically recover (confirmed by bacteriological culture), it was culled in the subsequent time period.

Probabilities of cure from clinical IMI, following treatment for the different treatment regimens, were obtained from Steeneveld et al. (2011) and are presented in Table 1 together with other input values. A clinical IMI case was considered a new case when at least $14 \mathrm{~d}$ had elapsed since the previous clinical IMI case (Halasa et al., 2009). Clinical flare ups, which are subclinical 
Table 1. Input parameter values ${ }^{1}$ for the different treatment regimens

\begin{tabular}{lccc}
\hline & \multicolumn{3}{c}{ Intervention scenario } \\
\cline { 2 - 4 } Parameter & $\begin{array}{c}\text { 3-d intramammary } \\
\text { treatment }\end{array}$ & $\begin{array}{c}\text { 5-d intramammary } \\
\text { treatment }\end{array}$ & $\begin{array}{c}\text { 5-d intramammary }+ \\
\text { 3-d systemic treatment }\end{array}$ \\
\hline Milk withdrawal (d) & 5 & 7 & 7 \\
Clinical IMI cure probability & 0.4 & 0.6 & 0.7 \\
Staphylococcus aureus & 0.7 & 0.8 & 0.9 \\
Streptococcus spp. & 0.8 & 0.8 & 0.8 \\
Escherichia coli & 15 & 25 & 1.08 \\
Total antibiotic costs $(€)$ & 0.75 & 1.03 & \\
Total treatment time $(\mathrm{h})$ & & &
\end{tabular}

${ }^{1}$ Values were obtained from Steeneveld et al. (2011).

cases that become clinical, were also considered new cases and treated according to the applied intervention scenario. No treatment was applied to subclinical IMI cows and their spontaneous cure rates were based on van den Borne et al. (2010b). Intramammary infection remissions were defined as clinical IMI cases that do not recover bacteriologically following treatment, but persist as subclinical IMI cases.

\section{Economic Analysis}

Costs of intervention depended on the applied scenario. In scenario 1 and 2, extra costs of treatment, labor time and milk withdrawal were added. In scenario 3 the extra costs included costs of bacterial culture (€19 for sampling and materials and $10 \mathrm{~min}$ of labor; van den Borne et al., 2010a) to confirm bacteriological cure of the clinical IMI cases, and the costs of culling. In scenario 4, extra costs including costs of treatment, labor time, milk withdrawal, bacterial culture, and culling costs. Calculation of the costs of labor and milk withdrawal is explained by Halasa et al. (2009). The input parameter values are presented in Table 1.

Costs of clinical and subclinical IMI during lactation were calculated as follows (Halasa et al., 2009). The sum of the cost of milk yield loss due to clinical IMI, costs of culling, costs of veterinary service, bacteriological culture costs, and costs of labor and antibiotic costs minus the saved cost, which is attributed to lower feed cost (Halasa et al., 2009), were calculated into the annual net cost of clinical IMI caused by the 4 simulated IMI pathogens. The annual net cost of subclinical IMI caused by the 4 simulated IMI pathogens was calculated as the sum of the costs of milk yield loss due to subclinical IMI, and costs of culling, minus the saved cost of feed.

The total annual net costs of IMI during lactation caused by the 4 simulated IMI pathogens were calculated as the sum of the annual net costs of clinical IMI, including intervention costs, and costs of subclinical
IMI. Costs were presented in Euros as average, and fifth and 95th percentiles to represent the economic variation. Numbers of IMI events were presented as medians. The number of IMI events and the total annual net costs of IMI in scenarios $1,2,3$, and 4 and the default scenario were compared with the estimate of the economic consequence of intervention against contagious clinical IMI.

\section{Parameter Variation}

Cows respond differently to antibiotic treatment of clinical IMI (Sol et al., 2000; Steeneveld et al., 2011), which makes it important to assess the epidemiologic and economic effects of changing the cure rates at fixed costs of treatment. Nonetheless, cure rate values in the current study were based on estimates by Steeneveld et al. (2011), which were actually the maximum likely values of pathogen-specific cure rates. Thus, only reduction of cure rate values by $25 \%$ was investigated for the simulated scenarios, in separate model runs. This reduction in cure rates would allow a realistic investigation of the effect of low cure rates of clinical IMI, as lower cure rates were observed (e.g., McDougall et al., 2007; Bradley and Green, 2009).

To study the economic consequence of interventions under different transmission rates compared with the default scenario, the pathogen-specific transmission rate parameter was changed one at a time in separate model runs. For contagious IMI pathogens, the transmission rate was changed, whereas the cumulative incidence was changed for E. coli. This was carried out for Staph. aureus by multiplying the transmission rate by 0.5 and $0.75 \%$ and by 1.25 and $1.5 \%$ in separate model runs.

The distribution of pathogens at the start was approximately 68\% Staph. aureus, 28\% Streptococcus spp., and $4 \%$ E. coli, representing a herd dominated by Staph. aureus (Halasa et al., 2009). It is interesting to study the economic consequence of interventions when Streptococcus spp. were the dominant pathogens. To 
do so, the distribution of pathogens at the start was changed to $11 \%$ Staph. aureus, $85 \%$ Streptococcus spp., and $4 \% \mathrm{E}$. coli.

Changes to economic input values focused on investigating the effect of prices changes to antibiotics and labor time for intervention of clinical IMI. The sensitivity of the model to other parameters was thoroughly studied previously (Halasa et al., 2009; Halasa et al., 2010; van den Borne et al., 2010a).

\section{Model Run and Stabilization}

Model run was carried out as explained previously (van den Borne et al., 2010a). It was run for 2 quota years, starting with the same herd situation per model run to ensure a stable infection process over time. The rate of pathogen-specific IMI at the end of the second quotum year was used to start a new model run for 2 quota years to be used for epidemiologic and economic assessment. The epidemiologic and economic output from the second quotum year only was used to conduct the assessment per scenario, to simulate the effect of the applied intervention in the herd and not the initial herd status (Østergaard et al., 2005). The model was replicated until the change in the total annual net cost of IMI changed by $<2 \%$, which was the case using 3,000 iterations. The model was iterated 1,000 times when model parameters were varied, because of less interest in extreme situations.

\section{RESULTS}

\section{Intervention Scenarios}

Results of herd characteristics and performance that are not related to IMI are presented in Halasa et al. (2009 and 2010) and did not differ between model scenarios. Per scenario, the annual cumulative incidence of total IMI, clinical and subclinical IMI cases, cured clinical IMI cases, culled clinical IMI cases due to intervention, and IMI remissions are presented in Table 2. The median annual cumulative incidence of IMI, when 3-d intramammary treatment was applied (default scenario), was 59, whereas the incidence using scenario 1 (5-d intramammary treatment) was 36 cases per year. In scenarios 2, 3, and 4, the median annual cumulative incidences were 30,26 , and 23 , respectively. The median annual cumulative incidence of clinical IMI in the default scenario was 30. The incidence was considerably lower in scenarios 1 through 4 , in which it was $21,18,16$, and 14 cases per year, respectively. Similarly, the annual cumulative incidence of subclinical IMI was considerably lower in scenarios 1 through 4 compared with the default scenario (Table 2). The default scenario showed a large variability in the number of IMI cases compared with the intervention scenarios (Table 2 ). This means that under these intervention scenarios, extreme situations are less likely to happen.

In the default scenario, the median number of recovered clinical IMI cases following treatment was 14 out

Table 2. Average, median, fifth, and 95th percentile of the number of clinical and subclinical IMI cases during lactation in 5 scenarios of clinical IMI intervention in a 100-cow dairy herd

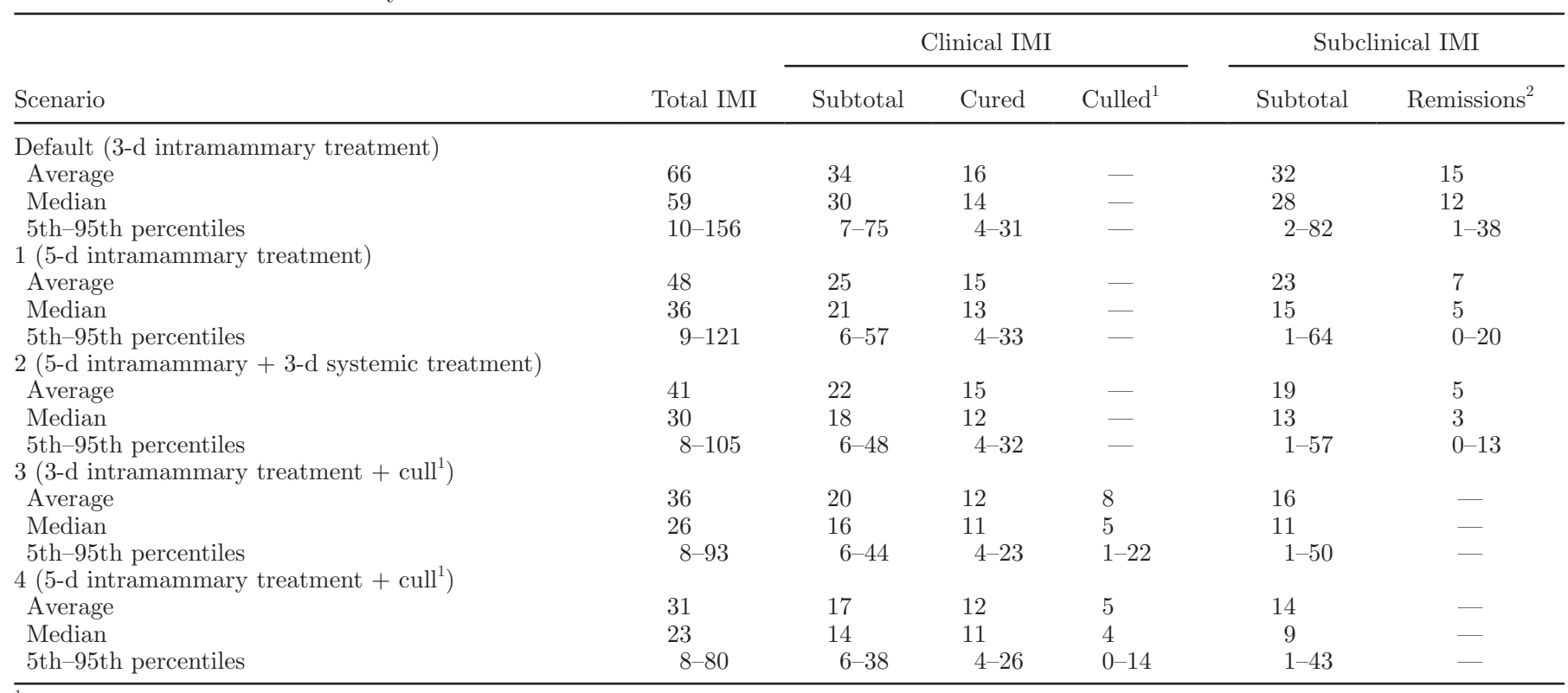

${ }^{1}$ Culling of treated and bacteriologically unrecovered clinical IMI cases (intervention culling).

${ }^{2}$ Originated from unrecovered clinical IMI case. 
Table 3. Average total annual net costs (fifth-95th percentiles) of clinical and subclinical IMI during lactation for 5 scenarios of clinical IMI intervention in a 100-cow dairy herd

\begin{tabular}{|c|c|c|c|c|c|}
\hline Cost factor $(€)$ & $\begin{array}{c}\text { Default scenario: } \\
\text { 3-d IT }\end{array}$ & Scenario 1: 5-d IT & $\begin{array}{l}\text { Scenario 2: 5-d } \\
\text { IT + 3-d ST }\end{array}$ & $\begin{array}{l}\text { Scenario 3: 3-d } \\
\text { IT }+ \text { cull }^{3}\end{array}$ & $\begin{array}{l}\text { Scenario } 4: 5 \text {-d } \\
\text { IT }+ \text { cull }\end{array}$ \\
\hline Clinical IMI & $3,037(332-7,030)$ & $2,650(415-6,412)$ & $2,832(545-6,665)$ & $4,858(778-13,334)$ & $4,196(946-8,093)$ \\
\hline Veterinary service & $253(53-563)$ & $187(45-428)$ & $162(45-360)$ & $146(45-330)$ & $129(45-285)$ \\
\hline Labor & $456(95-1,013)$ & $462(111-1,057)$ & $420(117-933)$ & $262(81-594)$ & $320(111-705)$ \\
\hline Culling & $1,789(0-4,520)$ & $1,352(0-3,516)$ & $1,148(0-3,014)$ & $3,840(502-11,050)$ & $2,922(0-8,538)$ \\
\hline Subclinical IMI & $2,929(1-8,046)$ & $1,927(1-6,033)$ & $1,557(0-5,030)$ & $1,158(0-3,526)$ & $986(0-3,019)$ \\
\hline Milk production & $158(7-400)$ & $110(6-314)$ & $91(5-274)$ & $73(5-222)$ & $63(4-205)$ \\
\hline Culling & $2,923(0-8,036)$ & $1,923(0-6,027)$ & $1,554(0-5,023)$ & $1,155(0-3,515)$ & $984(0-3,516)$ \\
\hline Saved costs & $152(6-385)$ & $106(5-303)$ & $88(5-264)$ & $70(5-214)$ & $61(4-198)$ \\
\hline Total net IMI costs & $5,966(689-14,221)$ & $4,577(572-11,776)$ & $4,389(701-10,971)$ & $6,016(823-16,664)$ & $5,182(872-14,071)$ \\
\hline
\end{tabular}

${ }^{1}$ Intramammary treatment.

${ }^{2}$ Systemic treatment.

${ }^{3}$ Culling of treated and bacteriologically unrecovered clinical IMI cases (intervention culling).

of 30 cases per year, whereas the median number was 13 out of 21 clinical IMI cases per year in scenario 1 . In scenarios 2 through 4 , the majority of clinical IMI cases would recover due to the intensive intervention (Table 2 ). In the default scenario and in scenarios 1 and 2, unrecovered clinical IMI cases would either be culled or more likely persist as subclinical IMI cases (IMI remissions). In the default scenario, out of the 30 clinical IMI cases per year, 12 persisted as subclinical IMI cases (Table 2). The median number of IMI remissions in scenarios 1 and 2 were 5 and 3 cases per year, respectively, showing the positive effect of long duration and intensive treatment of clinical IMI. In scenarios 3 and 4, IMI remissions would be culled, leading to a median number of culled clinical IMI cases due to intervention of 5 and 4 cases per year, respectively (Table 2). Scenario 4 showed a lower variability in the number of culled IMI remissions than scenario 3, due to the longer treatment duration.

The amount of antibiotics used differed between the scenarios. In the default scenario, the median number (with the fifth and 95th percentiles) of intramammary injections was 90 (21-225) per year. In scenario 1, 3 , and 4 , the values were 105 (30-285), 48 (18-132), and 70 (30-190) injections per year. In scenario 2, the median number of intramammary injections was $90(30-240)$ per year, but it also included 54 (18-144) systemic injections per year.

The average associated costs of clinical and subclinical IMI (together with the fifth and 95th percentiles) of the default and intervention scenarios are presented in Table 3. The average total annual net costs of IMI in the default scenario (3-d intramammary treatment) were $€ 5,966$, but varying from $€ 689$ to $€ 14,221$. The costs in scenarios 1, 2, and 4 were, respectively, €1,389,
$€ 1,577$, and $€ 784$ lower than the default scenario. Moreover, the variability was also lower in these scenarios (Table 3). On the other hand, scenario 3 (3-d treatment and culling of bacteriologically unrecovered cows) had slightly higher costs than the default scenario. The total annual net cost of IMI in scenario 3 was $€ 6,016$, and varied from $€ 823$ to $€ 16,664$. The higher costs and variability in this scenario are largely attributable to the high associated costs of culling clinical IMI cases (Table 3). Scenario 2 (5-d intramammary treatment + 3 -d systemic treatment) showed the lowest total annual net costs of IMI (Table 3). It also resulted in the lowest variability; the fifth and 95th percentiles were $€ 701$ and $€ 10,971$, respectively. Nonetheless, the difference between scenarios 1 and 2 was rather small.

\section{Parameter Variation}

The model was rather sensitive to reducing the probability of cure following antibiotic treatment of clinical IMI during lactation (Table 4). Reducing the probability of cure resulted in more new IMI cases, fewer recovered clinical IMI cases and, hence, more IMI remissions, and substantially larger economic damage (Table 4). Nonetheless, reducing the probability of cure did not change the ranking of the intervention scenarios. Scenario 2 (5-d intramammary treatment +3 -d systemic treatment) was still the most cost-effective, with slight difference from scenario 1 (5-d intramammary treatment; Table 4).

The results were sensitive to the changes in the transmission rate parameter of Staph. aureus (Figure 1). Moreover, the ranking of control strategies has consequently changed. For instance, scenario 2 (5-d intramammary +3 -d systemic treatment) was the most cost- 
Table 4. Median cumulative incidence of clinical and subclinical IMI and the average total annual net costs of IMI during lactation in a 100-cow dairy herd in 5 scenarios of clinical IMI intervention with a $25 \%$ reduction of the default probabilities of cure

\begin{tabular}{|c|c|c|c|c|c|}
\hline Cumulative IMI incidence & $\begin{array}{c}\text { Default scenario: } \\
\text { 3-d IT }\end{array}$ & $\begin{array}{l}\text { Scenario 1: } \\
5 \text {-d IT }\end{array}$ & $\begin{array}{c}\text { Scenario 2: } \\
\text { 5-d IT + 3-d ST }\end{array}$ & $\begin{array}{c}\text { Scenario 3: } \\
3 \text {-d IT }+ \text { cull }^{3}\end{array}$ & $\begin{array}{l}\text { Scenario 4: } \\
\text { 5-d IT + cull }\end{array}$ \\
\hline Total & 67 & 51 & 44 & 30 & 26 \\
\hline Culled due to intervention & 0 & 0 & 0 & 9 & 7 \\
\hline Subclinical & 37 & 24 & 19 & 12 & 11 \\
\hline Remissions & 21 & 12 & 9 & 10 & 7 \\
\hline
\end{tabular}

${ }^{1}$ Intramammary treatment.

${ }^{2}$ Systemic treatment.

${ }^{3}$ Culling of treated and bacteriologically unrecovered clinical IMI cases (intervention culling).

effective scenario using the default transmission rate of Staph. aureus. This was also the case at a Staph. aureus transmission rate of 1.5 times the default value, despite resulting in a larger number of IMI cases than scenario 4 (Figure 1). Nonetheless, when the transmission rate of Staph. aureus was 0.5 times the default value, the default scenario was the most cost-effective scenario, despite having the largest number of IMI cases. The total annual net costs of IMI at a low transmission rate of Staph. aureus for the default scenario were $€ 1,867$ and for scenario 1 through 4 were $€ 1,903$, $€ 1,946$, €2,531, and $€ 2,719$, respectively. Combining antibiotic treatment to culling of bacteriologically unrecovered clinical IMI cases was not economically beneficial at 1.5 times the default transmission rate of Staph. aureus (Figure 1). At this high transmission rate, intensive antibiotic treatment (scenarios 1 and 2) could limit the losses compared with the other scenarios, but large economic damage could not be avoided (Figure 1). The model was less sensitive to changes to the transmission rate of the Strep. uberis and Strep. dysgalactiae and to the cumulative incidence of $E$. coli (results not shown).

When the prevalence of the pathogens was changed in which the modeled contagious Streptococcus spp. dominated the herd, scenarios 1 and 2 became equally good in controlling clinical IMI cases (Figure 2). Nonetheless, scenario 2 seems to be slightly better in preventing extreme losses, as it has the lowest maximum value. Changing the economic parameters had a small and negligible effect on the model results and the ranking of the different scenarios did not change and, thus, these results are not further shown.

\section{DISCUSSION}

The indirect effects of modeling the dynamics of spread of contagious IMI pathogens on the total annual net costs of IMI during lactation using different intervention scenarios against contagious clinical IMI was investigated in the current study as recommended by Steeneveld et al. (2011). The results suggest that interventions against clinical IMI cases of contagious origin including long-duration antibiotic treatment can be economically beneficial. In the default scenario (3-d intramammary treatment), $40 \%$ of the treated clinical IMI cases persisted (IMI remissions) as subclinical IMI cases (based on median numbers from Table 2). These cows would act as a constant source of infection to herd mates, leading to a persistent IMI problem within the herd, and, thus, large economic damage (Table 3). When a longer duration and more intensive treatment was used in scenarios 1 and 2, only 24 and $17 \%$ of the clinical cases, respectively, persisted as subclinical IMI cases. The higher cure rates in these scenarios clearly resulted in a lower number of chronic IMI cows and, hence, lower risk of infection transmission to healthy herd mates. The higher cure rates and the lower risk of infection transmission in scenarios 1 and 2 paid off the higher costs of treatment and resulted in lower annual net costs of IMI during lactation compared with the default scenario. Furthermore, scenarios 1 and 2 showed the lowest variability in the total annual net costs of IMI (Table 3). This is also important, as some farmers are risk averse and, thus, a scenario with the lowest variability would be more attractive. The current study showed similar results to those of van den Borne et al. (2010a) who showed that lactational treatment of contagious subclinical IMI cows resulted in a large economic profit due to limiting transmission of infection between cows. Pinzón-Sánchez et al. (2011) estimated the economic consequence of short and long duration treatment of clinical IMI using a decision tree model. They found that short-duration treatment (2d) was the cheapest choice in case of contagious and environmental clinical IMI cases. The authors included transmission of IMI at the cow level and only from clinical cases. In case of IMI transmission, subclinical cases are perhaps more problematic than the clinical 

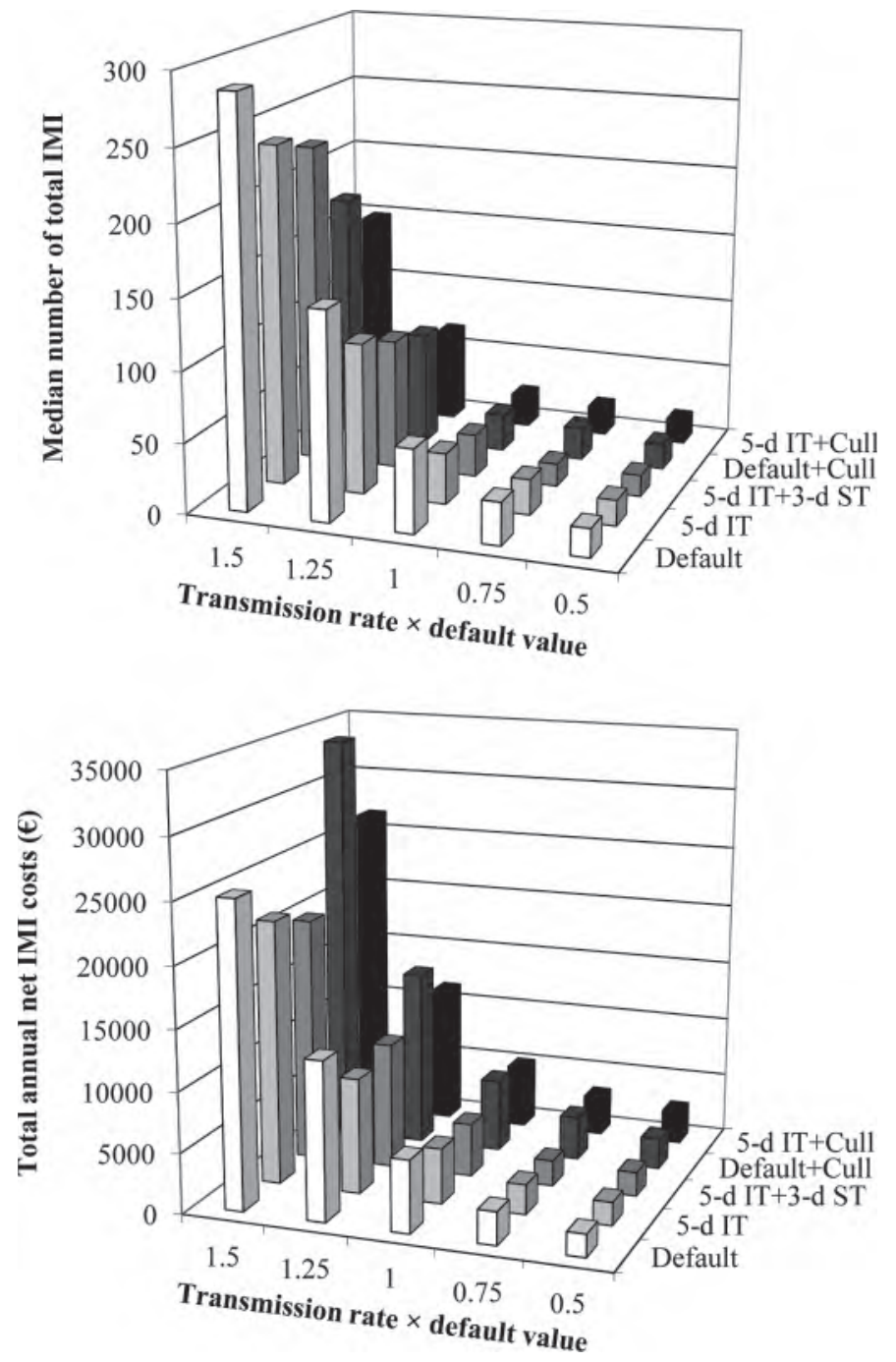

Figure 1. Median annual cumulative incidence of IMI (top) and average total annual net costs of IMI during lactation (bottom) in a 100-cow dairy herd with $0.5,0.75,1,1.25$, and 1.5 times the default transmission rate value of Staphylococcus aureus in the default scenario with 3-d intramammary treatment (IT); scenario 1 with 5-d IT; scenario 2 with 5-d IT and 3-d systemic treatment (ST); scenario 3, which included the default scenario and culling bacteriologically unrecovered clinical IMI cases (Cull); and scenario 4, which included scenario 1 and culling bacteriologically unrecovered clinical IMI cases.

cases, because they are invisible source of infection and could persist much longer than the clinical cases. In addition, the reduction of infected cows through treatment over time, combined with an increasing number of susceptible cows and the other aspects of transmission dynamics, were not modeled. These elements are important to reflect the dynamics of contagious IMI spread between cows at the herd level (Halasa et al., 2009; van den Borne et al., 2010a). To illustrate the importance of modeling transmission of infection, Staph. aureus spread was built in a Greenwood model with a

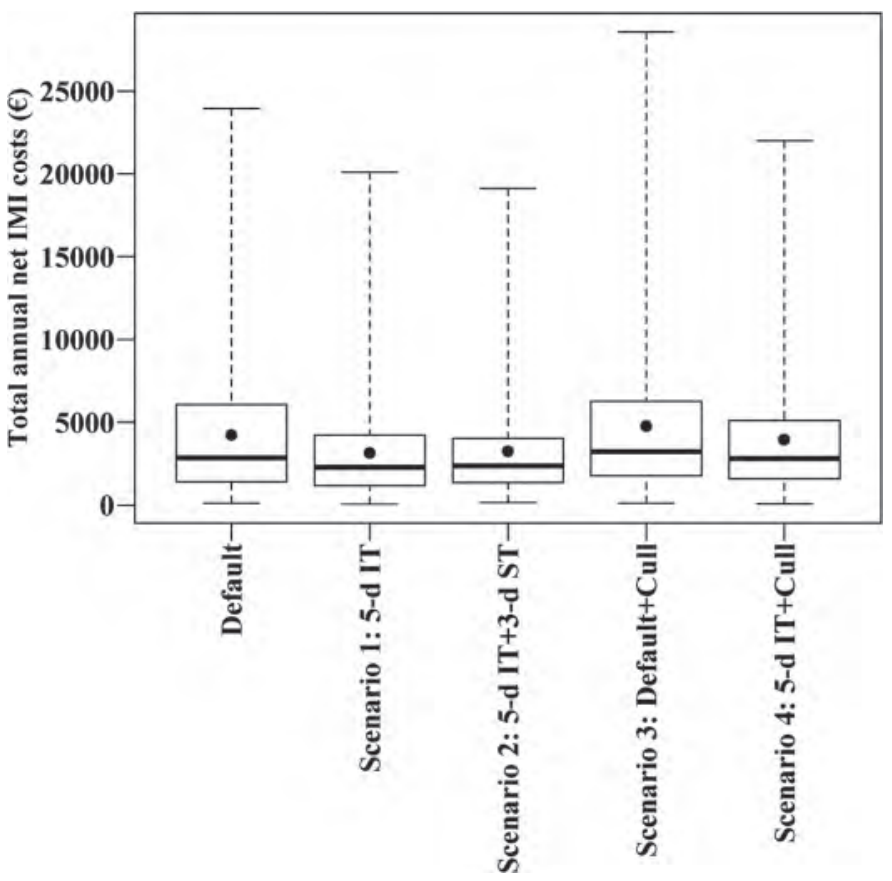

Figure 2. Box plot of the total annual net costs of IMI when the distribution of pathogens at the start was $11 \%$ Staphylococcus aureus, 85\% Streptococcus spp., and 4\% Escherichia coli., using the default scenario with 3 -d intramammary treatment (IT); scenario 1 with 5-d IT; scenario 2 with 5-d IT and 3-d systemic treatment (ST); scenario 3 , which included the default scenario and culling bacteriologically unrecovered clinical IMI cases (Cull); and scenario 4, which included scenario 1 and culling bacteriologically unrecovered clinical IMI cases. The box represents the 25 th, 50 th, and 75 th percentiles, whereas the whiskers represent the minimum and maximum values. The dark circle represents the average value.

cumulative incidence of $2 \%$ per time step and restricting the spread of the other pathogens. This resulted in an average total annual net cost of IMI of $€ 6,279$ (€3,648-€9,201) using the default scenario, and $€ 6,698$ (€4,105-€9,680) using scenario 2. Therefore, previous studies that estimated the effect of intervention against contagious clinical IMI during lactation, such as long duration antibiotic treatment, seem to have underestimated the true effect of the intervention, because of ignoring modeling the transmission of contagious IMI pathogens between cows at the herd level.

Combining culling with intramammary treatment, as in scenario 3 and 4 , would mean that the risk of IMI remissions is eliminated and, thus, limited disease transmission within the herd would occur. Scenario 3 has, however, resulted in very high costs of culling of clinical IMI, which made it more expensive than the default scenario (Table 3). Interestingly, scenario 4 showed considerably lower costs than the default scenario. The 5-d intramammary treatment would ensure a sufficiently high cure rate and fewer culled cows due to intervention (compared with scenario 3 ; see Table 2) 
and, thus, lower culling costs of clinical IMI, making this scenario cheaper than the default scenario (Table $3)$. Nonetheless, this scenario was more expensive than scenarios 1 and 2 , in which only antibiotic treatment was applied. These results are in accordance with findings of Heikkilä et al. (2012) who showed that it was mainly profitable to treat clinical IMI cows. A scenario in which clinical IMI cows were culled before treatment was not simulated, because such a scenario is not realistic (Steeneveld et al., 2011).

Scenario 2 (5-d intramammary +3 -d systemic treatment), seems to be the optimal scenario to minimize the losses due to IMI (Table 3). Nonetheless, this scenario would have included the use of the largest amount of antibiotics compared with the other scenarios. Recent concerns regarding antimicrobial resistance encourage minimizing the use of antibiotics in livestock production systems and, thus, a balance between antibiotics use and economic profit should be found. In scenario 4 (5-d intramammary treatment + culling bacteriologically unrecovered cows), a smaller amount of antibiotics (per year) would have been used and minimal economic damage would have been observed; compared with the default scenario. Therefore, this scenario can be recommended, as it has the potential to reduce antibiotics usage and still provide economic profit, compared with the default scenario.

Reducing the cure rates of clinical IMI resulted in substantial economic damage, due to the larger number of remission IMI cases (compared with the default cure rate values) and, hence, a wider spread of IMI within the herd (Table 4). Cows respond differently to antibiotic treatment (Sol et al., 2000; Steeneveld et al., 2011) and, thus, cow-specific treatment was suggested to optimize decision making regarding clinical IMI and, hence, productivity (Barkema et al., 2006; Steeneveld et al., 2011). Cow-specific treatment was not possible in the current model structure and, thus, future research regarding decision making on clinical IMI should combine modeling the dynamics of IMI transmission together with cow-specific decisions, such as treatment regimens and culling.

Little quantitative knowledge is available on the transmission rate of contagious IMI pathogens (van den Borne et al., 2010a). The pathogen-specific transmission rate parameter estimates in the current model were obtained from Zadoks et al. (2001, 2002). Due to differences between herds in management and differences between IMI strains, it was important to investigate the effect of the simulated scenarios at different transmission rates. The model was highly sensitive to changes in the transmission rate of Staph. aureus. The ranking of the scenarios have consequently changed (Figure 1). Long and intensive treatment of clinical IMI cows was the most cost-effective strategy at default and high transmission rate of Staph. aureus IMI. This was not the case at low transmission of Staph. aureus, in which the default scenario (3-d intramammary treatment) was the most cost effective. This may be explained by the limited transmission of infection between cows, in which IMI cows did not pose a high risk to healthy herd mates. Thus, the high costs of long-duration and intensive treatment were not paid off by the reduction in the risk of IMI transmission following the treatment. It is important to notice that despite the positive effect of long and intensive antibiotic treatment of clinical IMI cases, the treatment was not capable of preventing large economic damage at high transmission rate of Staph. aureus (Figure 1). Therefore, treatment should be preceded by control strategies (extra hygiene, for instance) to reduce the high transmission rate. This is in accordance with findings of Barlow et al. (2009) and van den Borne et al. (2010a) who studied the effectiveness of lactational treatment of subclinical IMI. They found that treatment did not stop the fast spread of infection between cows at high transmission rate. Lam et al. (1996) showed that hygiene measures such as postmilking teat dipping can reduce the transmission of Staph. aureus between cows.

When the distribution of pathogens at start was changed, in which Streptococcus spp. predominated the herd, similar trends (Figure 2) were observed as when Staph. aureus was the predominant pathogen (Table 2 ). This indicates that regardless of which contagious IMI pathogen dominates the herd, the default scenario is not sufficient to minimize losses. In addition, further actions are needed to minimize the losses by, for instance, using long-duration antibiotic treatment with or without culling bacteriologically unrecovered clinical IMI cows. It was also observed that scenarios 1 and 2 became equally effective in minimizing losses. This can be explained by the higher cure rate (Table 1) of Streptococcus spp. and the lower transmission rate, compared with Staph. aureus (Halasa et al., 2009).

The current bioeconomic model simulated a contagious spread of Staph. aureus, Strep. uberis, Strep. dysgalactiae, whereas an environmental spread of $E$. coli was simulated. In real life, this simplification of reality might not be completely correct, as some strains could behave contagious and others environmental (Zadoks et al., 2000, 2003). Therefore, the results of the current study are a good application for herds with a predominant contagious IMI problem and occasional occurrence of environmental IMI cases. To represent herds with predominant environmental IMI problem in the current model, the transmission rate of contagious IMI pathogens was set to zero and the cumulative incidence of $E$. coli was doubled. The results showed that 
the default scenario was the most cost-effective choice, which is consistent with previous findings (e.g., PinzónSánchez et al., 2011; Steeneveld et al., 2011).

This bioeconomic model simulates a herd of dairy cattle in a system where a milk quota is applied (Halasa et al., 2009). The milk quota that is applied in European Union countries is expected to be abolished soon. This is expected to result in higher costs of milk withdrawal and milk production loss due to IMI (Østergaard et al., 2005), which makes it important to invest in eliminating contagious clinical IMI cases by, for instance, long-duration antibiotic treatment.

In the current bioeconomic model, no costs of culturing were added to diagnose the causative agent of the clinical IMI before treatment to find out whether the pathogen was contagious or not. Bacterial culture results would arrive late, whereas the treatment should be implemented immediately and, thus, exclude culture costs before treatment would be realistic. Furthermore, models were developed that can help in diagnosis of the IMI causative agent (Steeneveld et al., 2011). Nonetheless, adding extra culture costs to diagnose the causative agent of the clinical IMI cases would not change the conclusions.

Several drawbacks exist in the current model that were presented and discussed previously (Halasa et al., 2009), including model validation and comparison to other bioeconomic models. Of these drawbacks, cow factors such as parity, DIM, and repeated clinical cases did not affect the probability of infection, because transmission rates of contagious IMI pathogens were not available per factor strata. Furthermore, penalty due to high bulk tank SCC would most often be 0, because of the way SCC of healthy cows was modeled (Halasa et al., 2009). This could have resulted in underestimation of the effect of the intervention scenarios in the current study, especially in the default scenario and scenario 1 , which had the largest number of subclinical IMI cases (Table 2).

The current study focuses on defining cost-effective interventions against clinical IMI cases, taking into account the dynamics of contagious IMI pathogens. Future research should focus on defining cost-effective interventions against both clinical and subclinical IMI, as their occurrences are not independent from each other.

\section{CONCLUSIONS}

Using a bioeconomic simulation model, long duration, and intensive antibiotic treatment of contagious clinical IMI resulted in fewer IMI cases, IMI remissions, and low transmission of contagious IMI pathogens between cows. Furthermore, the total annual net costs of IMI during lactation were lowest when a 5-d intramammary treatment was combined with a 3 -d systemic treatment of clinical IMI cases. Nonetheless, this scenario would result in using the largest amount of antibiotics to treat clinical IMI cases. Treatment of clinical IMI cases should be preceded by interventions to limit the transmission of contagious IMI pathogens between cows.

\section{ACKNOWLEDGMENTS}

The author acknowledges Bart van den Borne (University of Bern, Bern, Switzerland) and Wilma Steeneveld (Wageningen University, Wageningen, the Netherlands) for the critical feedback on a previous version of the manuscript.

\section{REFERENCES}

Bar, D., L. W. Tauer, G. Bennett, R. N. Gonzalez, J. A. Hertl, Y. H. Schukken, H. F. Schulte, F. L. Welcome, and Y. T. Grohn. 2008. The cost of generic clinical mastitis in dairy cows as estimated by using dynamic programming. J. Dairy Sci. 91:2205-2214.

Barkema, H. W., Y. H. Schukken, and R. N. Zadoks. 2006. Invited review: The role of cow, pathogen, and treatment regimen in the therapeutic success of bovine Staphylococcus aureus mastitis. J. Dairy Sci. 89:1877-1895.

Barlow, J. W., L. J. White, R. N. Zadoks, and Y. H. Schukken. 2009. A mathematical model demonstrating indirect and overall effects of lactation therapy targeting subclinical mastitis in dairy herds. Prev. Vet. Med. 90:31-42.

Becker, N. G. 1989. Analysis of Infectious Disease Data. Chapman and Hall, London, UK.

Bradley, A. J., and M. J. Green. 2009. Factors affecting cure when treating bovine clinical mastitis with cephalosporin-based intramammary preparations. J. Dairy Sci. 92:1941-1953.

Broom, D. M. 1991. Animal welfare: Concepts and measurements. J. Anim. Sci. 69:4167-4175.

Dohoo, I. R., S. W. Martin, and H. Stryhn. 2003. Veterinary Epidemiologic Research. Atlantic Veterinary College Inc., Charlottetown, Prince Edward Island, Canada.

Halasa, T., K. Huijps, O. Østerås, and H. Hogeveen. 2007. Economic effects of bovine mastitis and mastitis management: A review. Vet. Q. 29:18-31.

Halasa, T., M. Nielen, R. B. M. Huirne, and H. Hogeveen. 2009. Stochastic bio-economic model of bovine intramammary infection. Livest. Sci. 124:295-305.

Halasa, T., M. Nielen, T. van Werven, and H. Hogeveen. 2010. A simulation model to calculate costs and benefits of dry period interventions in dairy cattle. Livest. Sci. 129:80-87.

Heikkilä, A.-M., J. I. Nousiainen, and S. Pyörälä. 2012. Costs of clinical mastitis with special reference to premature culling. J. Dairy Sci. 95:139-150.

Lam, T. J. G. M., M. C. M. Dejong, Y. H. Schukken, and A. Brand. 1996. Mathematical modeling to estimate efficacy of postmilking teat disinfection in split-udder trials of dairy cows. J. Dairy Sci. 79:62-70.

McDougall, S., D. G. Arthur, M. A. Bryan, J. J. Vermunt, and A. M. Weir. 2007. Clinical and bacteriological response to treatment of clinical mastitis with one of three intramammary antibiotics. N. Z. Vet. J. 55:161-170.

Østergaard, S., M. G. G. Chagunda, N. C. Friggens, T. W. Bennedsgaard, and I. C. Klaas. 2005. A stochastic model simulating pathogen-specific mastitis control in a dairy herd. J. Dairy Sci. 88:4243-4257.

Pinzón-Sánchez, C., V. E. Cabrera, and P. L. Ruegg. 2011. Decision tree analysis of treatment strategies for mild and moderate cas- 
es of clinical mastitis occurring in early lactation. J. Dairy Sci. 94:1873-1892.

Sol, J., O. C. Sampimon, H. W. Barkema, and Y. H. Schukken. 2000. Factors associated with cure after therapy of clinical mastitis caused by Staphylococcus aureus. J. Dairy Sci. 83:278-284.

Steeneveld, W., T. van Werven, H. W. Barkema, and H. Hogeveen. 2011. Cow-specific treatment of clinical mastitis: An economic approach. J. Dairy Sci. 94:174-188.

van den Borne, B. H. P., T. Halasa, G. van Schaik, H. Hogeveen, and M. Nielen. 2010a. Bioeconomic modeling of lactational antimicrobial treatment of new bovine subclinical intramammary infections caused by contagious pathogens. J. Dairy Sci. 93:4034-4044.

van den Borne, B. H. P., G. van Schaik, T. J. G. M. Lam, and M. Nielen. 2010b. Therapeutic effects of antimicrobial treatment during lactation of recently acquired bovine subclinical mastitis: Two linked randomized field trials. J. Dairy Sci. 93:218-233.
Zadoks, R., W. van Leeuwen, H. Barkema, O. Sampimon, H. Verbrugh, Y. H. Schukken, and A. van Belkum. 2000. Application of pulsed-field gel electrophoresis and binary typing as tools in veterinary clinical microbiology and molecular epidemiologic analysis of bovine and human Staphylococcus aureus isolates. J. Clin. Microbiol. 38:1931-1939.

Zadoks, R. N., H. G. Allore, H. W. Barkema, O. C. Sampimon, Y. T. Gröhn, and Y. H. Schukken. 2001. Analysis of an outbreak of Streptococcus uberis mastitis. J. Dairy Sci. 84:590-599.

Zadoks, R. N., H. G. Allore, T. J. Hagenaars, H. W. Barkema, and Y. H. Schukken. 2002. A mathematical model of Staphylococcus aureus control in dairy herds. Epidemiol. Infect. 129:397-416.

Zadoks, R. N., B. E. Gillespie, H. W. Barkema, O. C. Sampimon, S. P. Oliver, and Y. H. Schukken. 2003. Clinical, epidemiological and molecular characteristics of Streptococcus uberis infections in dairy herds. Epidemiol. Infect. 130:335-349. 